MATEC Web of Conferences 43, 01005 (2016)

DOI: $10.1051 /$ matecconf $/ 20164301005$

C) Owned by the authors, published by EDP Sciences, 2016

\title{
Ultrasonic Measurement of Elastic Modulus of Kelvin Foam
}

\author{
Sukwon $\mathrm{Oh}^{1}$, Jongchul $\mathrm{Oh}^{1}$ and Nohyu Kim²,a \\ ${ }^{1}$ Undergraduate School of Mechatronics Engineering, Korea University of Technology and Education, South Korea \\ ${ }^{2}$ School of Mechatronics Engineering, Korea University of Technology and Education, South Korea
}

\begin{abstract}
Elastic modulus of 3D-printed Kelvin foam plate is investigated by measuring the acoustic wave velocity of $1 \mathrm{MHz}$ ultrasound. An isotropic tetrakaidecahedron foam of $3 \mathrm{~mm}$ unit cell is designed and printed layer upon layer to fablicate a Kelvin foam plate of $14 \mathrm{~mm}$ thickness by 3D CAD/printer using ABS plastic. The Kelvin foam plate is filled completely with paraffin wax for impedance matching, so that acoustic wave may propagate through the porous foam plate. The acoustic wave velocity of the foam plate is measured using the time-of-flight (TOF) method to calculate the elastic modulus of the Kelvin foam plate based on acousto-elasticity.
\end{abstract}

\section{Introduction}

Three-dimensional cellular materials are widely used in the energy and transport industries as lightweight structural materials to achieve substantial gains in terms of increasing heat transfer, mixtures and chemical reactions. This cellular material is primarily used for its mechanical properties especially in the aerospace and transportation where lightweight and resistant structures are needed. The thermal properties of such materials are equally important in many targeted applications e.g. cooling of electronic components, thermal insulation (sandwich panels). These materials can provide simultaneously a set of excellent qualities e.g. mechanical, thermal, catalytic that lead to significant reductions in cost, weight and offer opportunities for applications in diverse areas including automobiles, fuel cells, chemical engineering [1]. To a lesser degree, the acoustic properties are also used in applications such as vibration damping, adjusting the sound and noise reduction. Heat exchangers, reactors with foam as internals are one of the good examples of application. Their field of applications have increased substantially in recent years due to the properties already mentioned. For example, porous foams attract an interest as biomaterials for implantation purpose as the introduction of pores into the bulk material provides in-growth of living tissues and firm fixation in addition to reducing the alloy density $[2,3]$. It is a good candidate as a biomedical apparatus and implant device. Metallic foam structure has low enough value of elastic modulus which is much smaller than that of the bulk metal and comparable to that of human bone. Kim et al [4] produced highly porous foam by sintering $\mathrm{Ni}$-Ti shape memory alloy fibers. Their porosity value was about $80 \%$. In recent years, since 3 -dimensional printing technology has emerged, an additive production method to build

\footnotetext{
a Corresponding author: nykim@koreatech.ac.kr
}

metallic structure by liquid metal has been proposed. It will be possible in the near future to make a foam structure by 3-D printer if one has the design of the foam $[5,6]$.

The mechanical behavior of a cellular material such as Kelvin foam depends on its relative density, the properties of the cell wall material, and the cell geometry. The individual response of cells to a loading is also strongly influenced by their location in the material, for instance, deformation of cells located in the bulk, surrounded by other cells is more constrained comparing to cells located adjacent to stress-free boundaries. It is reported that if the length, width, and thickness of a foam sample are large enough to include many cells, the differences in cellular response to a macroscopic loading are averaged out, leading to size-independent effective properties. If, however, one of the structural dimensions contains only a few cells, as is the case in many practical applications including this study, the effective response of the foam is dictated by the individual excitements of the cells, resulting in a size-dependent mechanical behavior.

In this work, the foam structure known as the Kelvin foam or tetrakaidecahedron is manufactured by 3D printer with $\mathrm{ABS}$ plastic material to investigte the Young's modulus of the foam structure by ultrasonic method. It is shown from experiment that the Young's modulus of the produced Kelvin foam is about $4 \%$ of the bulk value of the ABS plastic material, which agrees with theoretical results from FEM analysis and micromechanics model.

\section{Design of Kelvin structure}

Fig. 1 describes Kelvin foam microstructure. The cell is formed by both faces and edges; the cell edges are 
formed by the intersection of multiple faces. It has a 14sided polyhedron possessing eight hexagonal and six quadrilateral sides and packs to fill space. These cells are repeated periodically in the space so they can completely fill it. The unit cell structure is usually described by its morphological parameters, namely cell size, pore size, strut thickness and porosity.

The Kelvin cell considered in this study is described in Fig. 2, and Table 1. CAD image of the Kelvin cell is illustrated in Fig. 2(a) with a sample Kelvin foam structure made by 3D printer in Fig. 2(b) which consists of two layers of 16 open cells with $81 \%$ of porosity.

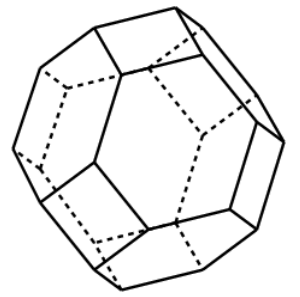

(a)

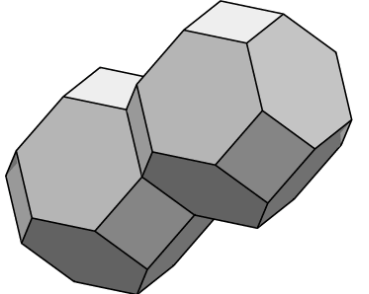

(b)

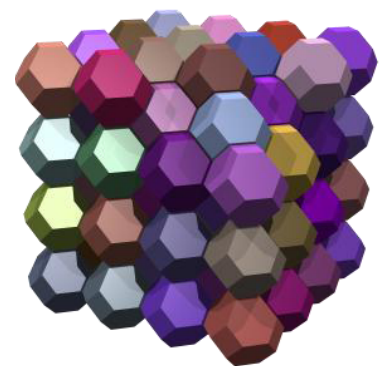

(c)

Figure 1. Kelvin structure, (a) unit cell, (b) 1-D, and (c) 3-D formation

Table 1. Dimensions of Kelvin cell edges
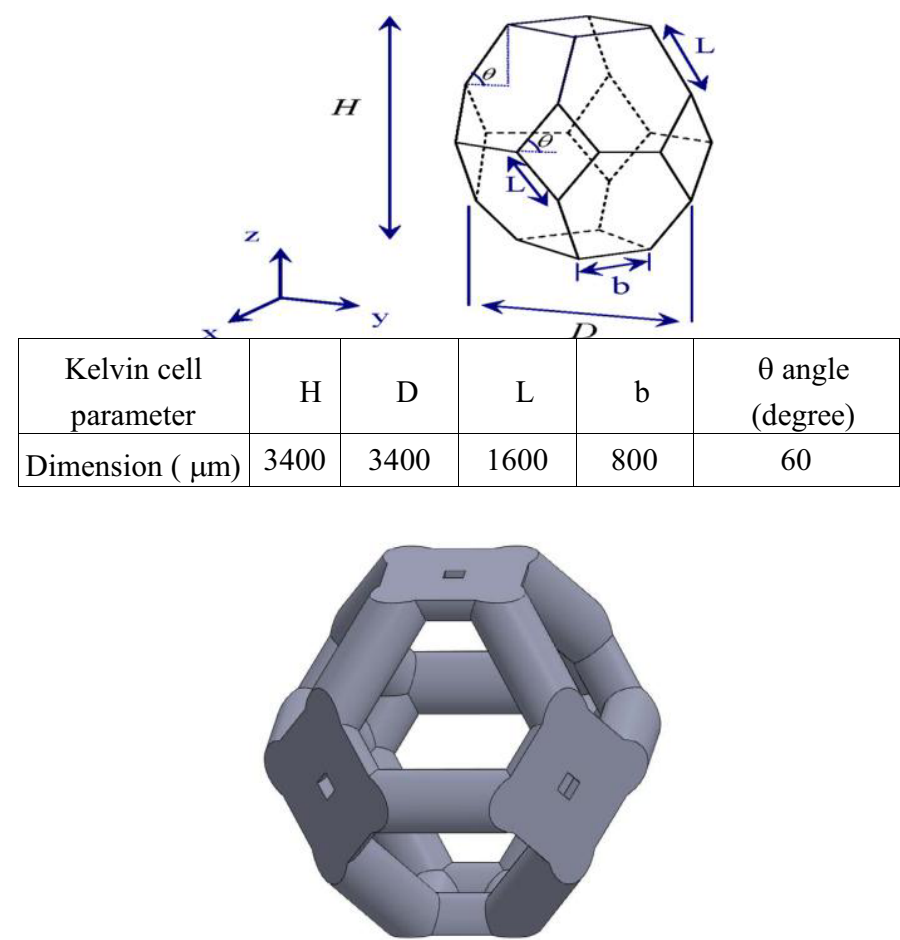

(a)

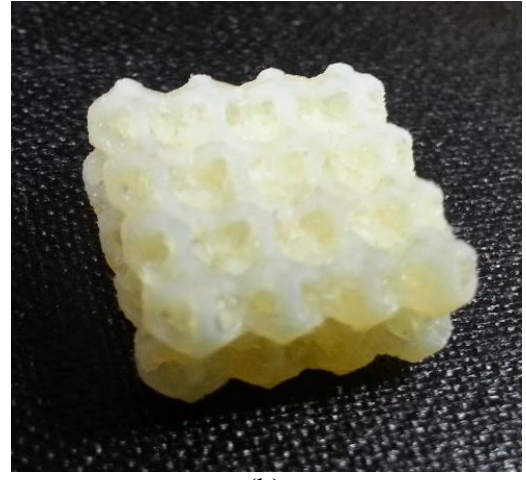

(b)

Figure 2. CAD image of the Kelvin cell and a 3D-printed example

For ultrasonic measurement, four layers of the Kelvin cells are printed layer upon layer by $3 \mathrm{D}$ printer to produce a $14 \mathrm{~mm}$-thick foam plate $(50 \mathrm{~mm}$ wide and $100 \mathrm{~mm}$ long) as shown in Fig. 3(a). As shown in Fig. 2(b) and Fig.3, the Kelvin foam plate designed in this study has so many empty space ( $81 \%$ of porosity), that the ultrasound cannot penetrate at all. Therefore, it is filled with paraffin wax to make a mixture of Kelvin structure with paraffin wax as in Fig. 3(b). The paraffin wax in the mixture works as a filler to print and support the Kelvin foam, but also to match the acoustic impedance between the Kelvin foam and empty pores. Fig. 3(a) represents the Kelvin cell structure itself, and Fig. 3(b) shows the two phase mixture, a Kelvin foam infiltrated with the paraffin wax.

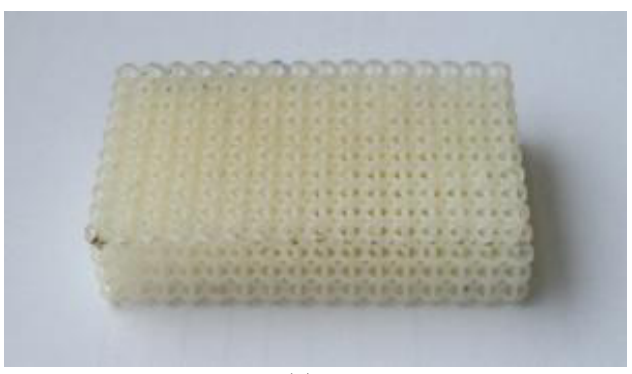

(a)

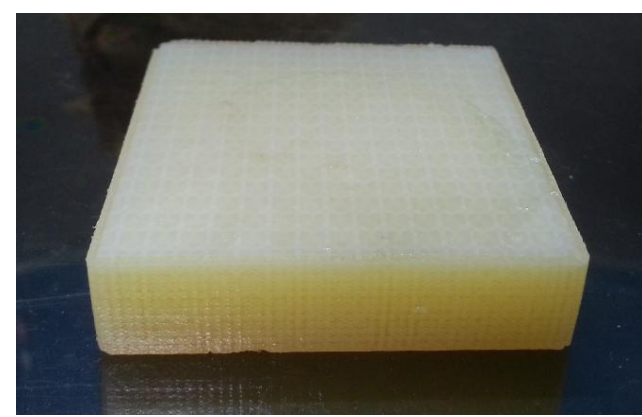

(b)

Figure 3. Kelvin foam plate by 3D printer, (a) Kelvin foam itself, (b) two phase mixture of Kelvin foam and paraffin wax

\section{Measurement of Elastic Modulus}

Measurements of ultrasonic velocities are conducted to estimate the elastic modulus of 3D-printed Kelvin 
structure. In the present work, the ultrasonic velocity measurements were carried out by using pulse-echo method, which uses the time-of-flight (TOF) measurement to calculate the wave velocity. In the TOF method, acoustic transducer generates a pulse signal that propagates through the structure and is reflected back, being detected after a delay. The duration of the wave propagation from the front surface to the back surface of the Kelvin structure depends on the thickness of the structure as well as on the elastic properties of the traveling medium (Kelvin structure). For an isotropic material, the elastic modulus is related to the ultrasonic wave velocity (compression mode) $c$ as following.

$$
E=\rho c^{2}
$$

where E is the Young's modulus, is the density of material. The configuration of ultrasonic measurement is set up as illustrated in Fig. 4(a) to measure the TOF of ultrasonic pulse inside the Kelvin foam plate. As stated above, the time of flight(TOF) of an ultrasonic pulse wave is evaluated from the front surface to the rear surface in a way illustrated in Fig.4(a). At the start of a measurement, a pulse generator excites a voltage pulse, which is converted in the ultrasonic transducer to an ultrasonic pulse which propagates through the plate at a velocity $c$. When it propagates, the pulse hits first the interface of the transducer and the Kelvin plate. After the pulse reflects at the back surface and returns to the active surface of the transducer, a voltage pulse appears at the amplifier output on oscilloscope screen, repeating this round-trip travel as shown in Fig. 4(a). When the amplitude of the received signals overcome a fixed threshold(i.e., noise level), the echoes are recorded, and the time interval between two maximum peaks of consecutive echoes is measured. Fig. 4(b) shows one example of measurement of the TOF based on this method for the bulk material of Kelvin foam, where the first pulse reflected on the front surface is too large to be seen completely because the second echo signal is much smaller than the first signal. This signal triggers counting the TOF of the ultrasonic wave. Fig. 4(b) shows one example of the measurement of the TOF for the Kelvin foam plate, where multiple echo signals from the back surface is captured and used to calculate the TOF of the ultrasonic wave. The acoustic velocity, $c$, is expressed through the TOF and the thickness of the plate, $d$, by the simple relation of Eq. (2)[7]

$c=2 d / \mathrm{TOF}$

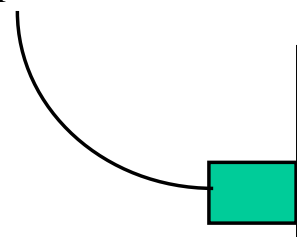

Ultrasonic transducer

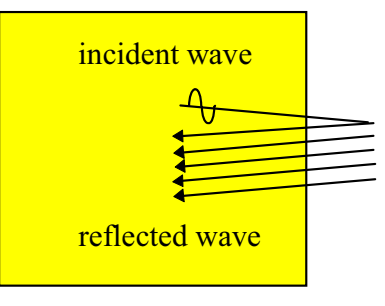

Kelvin foam plate

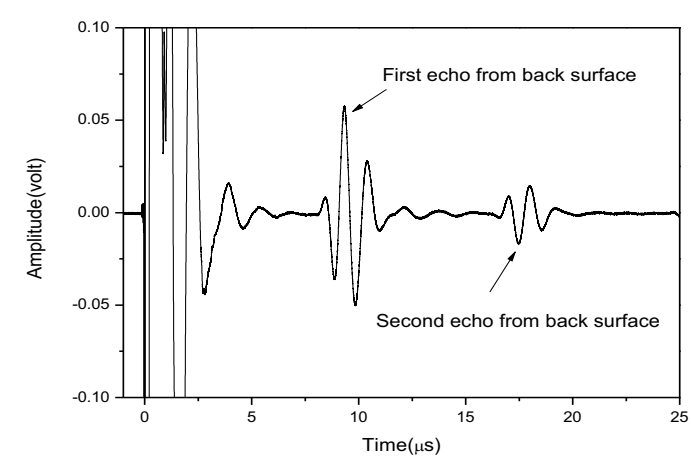

(b)

Figure 4. Wave propagation and reflection in Kelvin foam plate

If the Kelvin foam plate produced in Fig. 2(b) is assumed as a composite material, according to the elastic theory of composite materials, the elastic modulus of the two phase mixture, denoted by E, may be given by using the rule of mixtures[8]

$$
E=V_{K} E_{K}+V_{W} E_{W},
$$

where, $E_{W}$ is the elastic constant for paraffin wax, $E_{K}$ is the elastic constant for Kelvin foam, $V_{W}$ and $V_{K}$ are the volume fraction of wax and Kelvin foam.

Since the bulk material of Kelvin foam(ABS plastic) has a broad range of elastic property, its elastic modulus is determined experimentally by the same ultrasonic TOF method using a coin-type specimen of $100 \%$ bulk ABS plastic as shown in Fig. 5(a). The paraffin wax is also tested in the same way for accurate estimation of the elastic modulus of Kelvin foam. Fig. 5(b) displays the acoustic waveforms of $1 \mathrm{MHz}$ ultrasound acquired from the test specimens, which include two coin specimens of Fig. 5(a) and the mixture of Kelvin foam and wax in Fig. 3(b). Fig. 5(b) shows that the mixture has a wave speed between the bulk ABS plastic and the paraffin wax, but much closer to the wax's wave velocity. From the acoustic wave velocity obtained from Eq. (2) in ultrasonic test, the elastic modulus of the mixture is determined by Eq. (1) first. Table 2 lists the elastic and acoustic property of ABS plastic, paraffin wax, and the two phase mixture of Kelvin foam and wax. The elastic modulus of two phase mixture is compared in Table 3 with theoretical elastic modulus based on the rule of mixture derived from the mechanics of composite in Eq.(3). It shows a good agreement between the estimate of ultrasonic test and composite model for the mixture of the Kelvin foam and wax.

Table 2. Material property

\begin{tabular}{|c|c|c|c|c|}
\hline Material & $\begin{array}{c}\text { Density } \\
{\left[\mathrm{g} / \mathrm{cm}^{3}\right]} \\
\rho\end{array}$ & $\begin{array}{c}\text { Thickness } \\
{[\mathrm{mm}]} \\
d\end{array}$ & $\begin{array}{c}\text { Acoustic } \\
\text { velocity } \\
c[\mathrm{~mm} / \mathrm{sec}]\end{array}$ & $\begin{array}{c}\text { Elastic } \\
\text { modulus } \\
\mathrm{E}[\mathrm{GPa}]\end{array}$ \\
\hline ABS plastic & 1.15 & 10 & 2.40 & 6.63 \\
\hline Paraffin wax & 1.0 & 9.6 & 1.67 & 2.72 \\
\hline $\begin{array}{c}\text { Mixture (Kelvin } \\
\text { foam+wax) }\end{array}$ & 1.02 & 14 & 1.75 & 3.13 \\
\hline
\end{tabular}


The elastic modulus of two phase mixture is compared in Table 3 with theoretical elastic modulus based on the rule of mixture derived from the mechanics of composite in Eq.(3). It shows a good agreement between the estimate of ultrasonic test and composite model for the mixture of the Kelvin foam and wax.

The elastic modulus of two phase mixture is compared in Table 3 with theoretical elastic modulus based on the rule of mixture derived from the mechanics of composite in Eq.(3). It shows a good agreement between the estimate of ultrasonic test and composite model for the mixture of the Kelvin foam and wax.

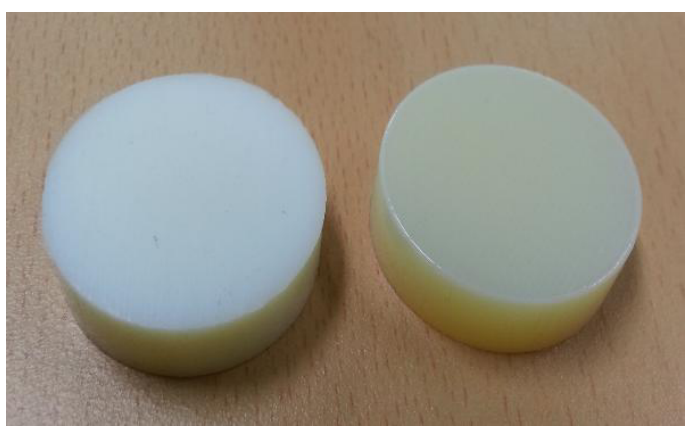

(a)

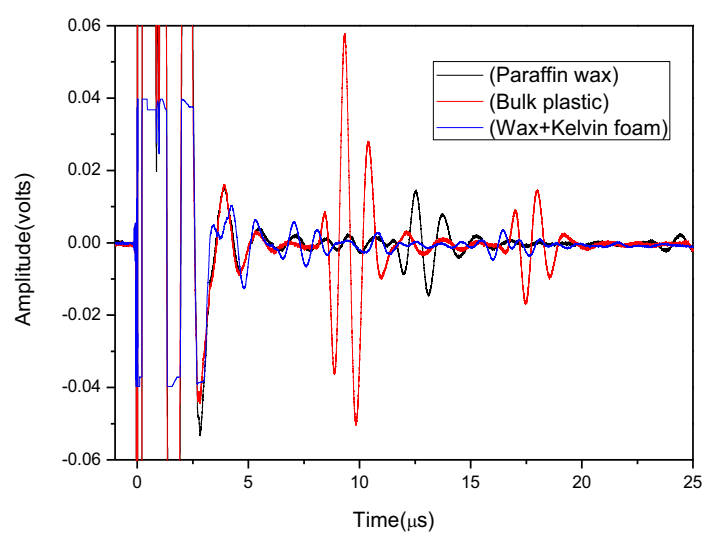

(b)

Figure 5. Shapes and waveforms of bulk ABS plastic and paraffin wax

Table 3. Elastic modulus of two phase mixture

\begin{tabular}{|c|c|c|}
\hline Result & $\begin{array}{l}\text { Elastic modulus of } \\
\text { Kelvin foam plate, } \\
\mathrm{E}_{\mathrm{K}}[\mathrm{MPa}]\end{array}$ & $\begin{array}{c}\text { Percentage of } \mathrm{E}_{\mathrm{K}} \text { with } \\
\text { respect to bulk material } \\
\mathrm{E}_{\mathrm{B}}, \mathrm{E}_{\mathrm{K}} / \mathrm{E}_{\mathrm{B}}(\%)\end{array}$ \\
\hline $\begin{array}{c}\text { Ultrasonic } \\
\text { test }\end{array}$ & 410 & 6.2 \\
\hline
\end{tabular}

Next, in order to extract the elastic modulus of the Kelvin foam from the elastic modulus of the mixture, a simple hypothesis is formed that the Kelvin structure and the wax work as two elastic springs connected in parallel as represented in Fig. 6. In Fig. 6, $E_{K}$ is the elastic modulus (spring constant) of the Kelvin foam and $E_{W}$ is the elastic modulus (spring constant) of the paraffin wax. On this assumption, the resultant elastic modulus (spring constant), $E$ of the mixture is equal to the simple sum of two elastic spring constants, i.e., $E=E_{K}+E_{W}$. Since $E_{W}$ and $E$ are known from Table 2, the elastic modulus of the Kelvin foam is easily determined by the equation $E_{K}=E-E_{W}$, which is $410 \mathrm{MPa}$ and $6.2 \%$ of the bulk ABS plastic material as listed in in Table 4.

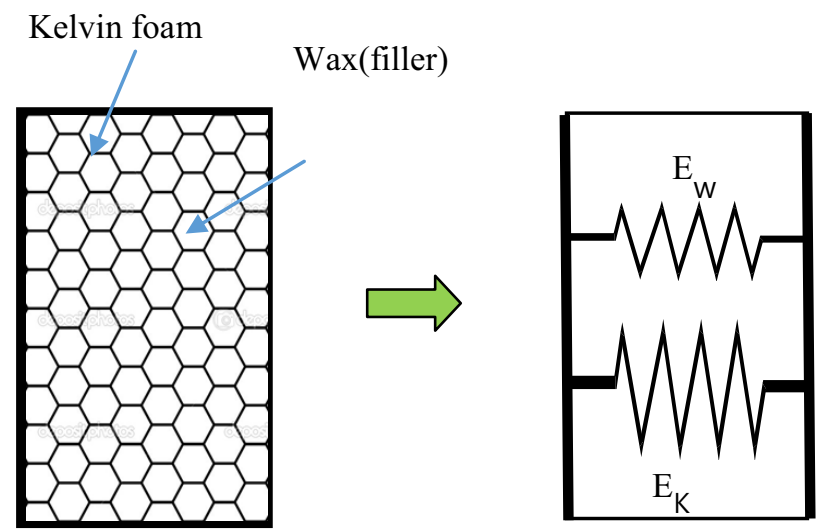

Figure 6. One-dimensional spring model of two phase mixture of Kelvin structure and wax

Table 4. Elastic modulus of Kelvin foam structure

\begin{tabular}{|c|c|}
\hline Method & $\begin{array}{c}\text { Elastic modulus of two phase } \\
\text { mixture[GPa] }\end{array}$ \\
\hline Ultrasonic test & 3.13 \\
\hline The rule of mixture & 3.46 \\
\hline
\end{tabular}

\section{Conclusions}

The elastic modulus of a 3D-printed Kelvin foam plate with $81 \%$ porosity is evaluated by the ultrasonic method using acoustic wave velocity based on TOF measurement. The porous Kelvin foam is infiltrated with paraffin wax to make two phase mixture of the Kelvin foam and to penetrate the acoustic wave. The mixture of Kelvin foam and wax is modeled as two linear springs connected in parallel, one of which is the Kelvin foam and the other the wax. From this simple spring model of the mixture, the elastic modulus of the Kelvin foam is estimated $410 \mathrm{Mpa}$ and $6.2 \%$ of the bulk material. It can be concluded from FEM and experiments results that the ultrasonic method is useful and effective to evaluate the elastic modulus of Kelvin foam structures in a fast and simple way. It is also expected that it has great potential for medical applications such as the measurement of bone density as well as industrial applications like industrial foams used for shock absorption and insulation.

\section{Acknowledgement}

This work was supported by Radiation Technology R\&D program through NSF funded by the Ministry of Science, ICT \& Future Planning (NRF-2013M2A2A9043274) and partly by University Research Program of Korea University of Technology and Education. 


\section{References}

1. P. Kumar, Ph. D thesis, Department of Mechanical Engineering, Aix-Marseille University, France (2014)

2. A. Ayers, S. J. Simske, T. A. Bateman, A. Petkus, R. L. C. Sachdeva and . E. Gyunter, Journal of Biomedical Materials Research, Vol. 45, No. 1, pp. 42-47 (1999)

3. G. Ryan, A. Pandit and D. P. Apatsidis, Biomaterials Vol. 27, No. 13, pp. 2651-2670 (2006)

4. Y-W Kim, T-H Nam and S. Young, Science of Advanced Materials, Vol. 6, pp. 2005-2009 (2014)
5. C. Prest, J. Poole, J. Stevick, T. Waniuk and Q Pham, , United States Patent Application Publication, No. US20130309121 (2013)

6. C. Tekoglu, L. Gibson, T. Pardoen, P. R. Onck, Progress in Materials Science, Vol. 56, pp. 109138(2011)

7. N. Kim and S. Lee, Journal of the Korean Society of Nondestructive Testing, Vol.23, No. 6, pp. pp. 577582(2003)

8. C. Chamis, Journal of Composites Technology and Research, Vol. 11, No. 1, pp. 3-14(1989) 